\title{
Music and dementia
}

This article was published in the following Dove Press journal:

Degenerative Neurological and Neuromuscular Disease

4 September 2013

Number of times this article has been viewed

\section{Balakrishnan R Nair' \\ William Browne ${ }^{2}$ \\ John Marley ${ }^{3}$ \\ Christian Heim ${ }^{4}$}

'University of Newcastle and the Centre for Medical Education, HNE Health, Newcastle, NSW, ${ }^{2}$ Geriatric Medicine, Eastern Health, Melbourne, VIC, ${ }^{3}$ Faculty of Health Sciences, The University of Queensland, Royal Brisbane and Women's Hospital, Brisbane, QLD, ${ }^{4}$ Toowong Private Hospital, Brisbane, QLD, Australia
Correspondence: Balakrishnan R Nair Continuing Medical Professional Development, School of Medicine and Public Health, Newcastle, NSW, Australia Email kichu.nair@newcastle.edu.au
Abstract: As the population ages, the prevalence of dementia is increasing. Distressing behavioral problems are often part of the illness. This review considers the available evidence for cognitive effects related to music, evidence for the efficacy of music in the management of behavioral problems in dementia, and evidence about the effects of different types of music, their mode of delivery, and any adverse effects. Live music may be more beneficial than recorded. The effect of music may not be lasting, but there is evidence of benefit in studies, which to date are mostly not of high quality.

Keywords: music, dementia, benefit

\section{Background}

The rising prevalence of dementia, allied closely with the aging of the population, poses distinct social, economic, and health care challenges. A recent epidemiologic assessment based on US census data estimated that, in 2010, there were 4.7 million individuals in that country aged 65 years or older with Alzheimer's disease (AD) dementia. ${ }^{1}$ The same study reported that the total number of people with AD dementia in the US in 2050 is projected to be 13.8 million. A similar pattern has been observed in other developed and developing countries; dementia is a global issue. ${ }^{2}$

Behavioral disturbances are common in dementia and lead to considerable stress for patients and caregivers. Typical disturbed behaviors include psychosis and paranoia, aggression, wandering, disinhibited behavior, and sleep disturbance. Managing these behaviors can be difficult, with increasing care needs and stress for all involved. The outcome may be residential placement or elder abuse. ${ }^{3}$

Pharmacotherapy may be of limited benefit and its use is controversial. Medications used include antipsychotic agents, benzodiazepines, cholinesterase inhibitors, antiepileptics, and antidepressants. There is limited evidence supporting the efficacy of these agents and they are often associated with significant adverse effects.

Nonpharmacologic strategies include the use of regular patient routines, exercise programs, regular toileting, and modification of personal care strategies. For a significant number of patients, these strategies are either not effective or are only partially effective. Therefore, there is a pressing need to explore additional strategies for the management of behavioral problems.

The search for alternative strategies to manage the behavioral symptoms of dementia has led to interest in the use of arts-based therapies for patients with cognitive disorders. ${ }^{4}$ Decades of case-based reports suggest benefit from such interventions, 
although the quality of the available evidence is limited. A Cochrane review updated in 2010 identified ten studies of music therapy in the management of behavioral problems associated with dementia and found that there was insufficient evidence owing to the quality of the studies to support or discourage the use of music therapy in this context. ${ }^{5}$ Problems with available studies include small numbers of subjects, inadequate descriptions of study participants, imprecise data collection methods, high attrition rates, and insufficient statistical analysis. Despite this, there is evidence to support the efficacy of activity programs, music, behavior therapy, light therapy, caregiver education, and changes to the physical environment. ${ }^{6}$

The use of music therapy has been proposed to assist in the management of agitated behaviors. Such therapies consist of either playing music to calm patients with dementia and to improve cooperation with caregivers, or utilization of a trained therapist to lead patient participation in playing or listening to music. Advantages of such therapies include low cost and the presumed absence of adverse effects.

Some studies including music and physical therapy have shown modest cognitive benefits of music therapy in dementia. ${ }^{7}$ Live music may be more beneficial than prerecorded music. One small study of 32 subjects found that live interactive music had immediate and positive engagement effects in subjects with apathy, regardless of the severity of their dementia. Prerecorded music was nonharmful but less clearly beneficial. ${ }^{8}$ The effects of music on behavior in dementia appear short-lived in studies to date, relating only to the immediate period during which music is being played. ${ }^{9}{ }^{10}$ Another small study found that passively listening to a preferred music was effective in reducing measures of anxiety in a population of residential care patients with dementia. ${ }^{11}$ Furthermore, music can improve duration and quality of sleep in both healthy elderly subjects and older people with $\mathrm{AD}$ dementia. ${ }^{12,13}$

There is also evidence for music therapy, as opposed to simply listening to music, for the management of dementiarelated behavioral symptoms. A program of music therapy improved composite measures of such symptoms in a small population of 59 patients with $\mathrm{AD}$ dementia. ${ }^{14}$

Music may lower biological markers of stress in dementia patients. A small study evaluating music therapy in elderly patients with senile dementia found beneficial effects on chromogranin A, confirming the benefits of music. ${ }^{15}$ While a measure of behavioral symptoms was also benefitted in this study, the overall effect of music therapy on behavioral symptoms of dementia is not a simple one, and there is evidence that under some circumstances the effect can be adverse. ${ }^{9}$

\section{Cognition and music}

Children with music training demonstrated better verbal but not visual memory than did their counterparts without such training. When these children were followed up for a year, those who had started or continued music training demonstrated significant verbal memory improvement. ${ }^{16}$ These findings are similar to those for adults, ${ }^{17}$ and suggest that music training systematically affects memory processing in accordance with possible neuroanatomic modifications in the left temporal lobe. ${ }^{16}$ Magnetic resonance imaging studies in adults with a history of childhood music training (before age 12 years) identified enhanced size of the left planum temporale region, an area in the temporal lobe, which is associated with improved verbal but not visual memory function. ${ }^{17}$ The effects of music on brain development have suggested a possible clinically useful effect in the care of patients suffering from neurodegenerative diseases.

Music, whether played or listened to, has neurocognitive effects that have been detected in various populations. Studies utilizing functional magnetic resonance imaging have allowed identification of diffuse activation of regions throughout the brain in predictable patterns. ${ }^{18}$

Studies of this type identified activation of a network from auditory relay stations in the midbrain to high-level cortical brain structures related to working memory and attention, and beyond that to movement-planning areas in the cortex. The frontoparietal cortex, which regulates tasks related to attention and working memory, displayed consistent responses between individuals, principally in the right hemisphere, particularly Broca's and Geschwind's area counterparts in the right hemisphere. ${ }^{18}$

One study found evidence for relatively preserved knowledge of music in a musically untrained patient with semantic dementia and characteristic asymmetric anterior temporal lobe atrophy. ${ }^{19}$ This suggests that music is partly separable functionally and anatomically from other semantic domains.

The central diagnostic feature of $\mathrm{AD}$ is memory loss. There is encouragement from small studies that memory for music may be retained in some individuals. One such controlled study investigated this and found that, while not universal, memory for music is preserved in a proportion of people with $\mathrm{AD}$ while other memory is lost. ${ }^{20}$ The recognition of familiar or unfamiliar memories may be impaired, but the ability to play a musical instrument may be retained. ${ }^{21}$ 


\section{What type of music?}

Many studies attest to the effectiveness of individualized music therapy in patients with dementia. ${ }^{22-25}$ The use of one-to-one music therapy in dementia is limited in clinical practice by significant cost factors. Ambient music, whereby music is played through a sound system, can be used to modify clinical environments in a cost-effective way.

Previous studies have shown benefit to patients when ambient music is used in a variety of clinical settings. These include outpatient procedures, ${ }^{26,27}$ postoperative recovery, ${ }^{28}$ and geriatric care settings. ${ }^{29-31}$ The use of patient-preferred music where patients are given a selection of music from which to choose is problematic. Allowing patients, particularly in dementia units, to choose their own music or to agree upon any particular music would be logistically challenging and difficult. One study showed benefit to two patients exposed to preferred music or nonpreferred music using headphones, but the study had only three patients in total and observation times were limited to 20 minutes per day. ${ }^{32}$

Reminiscence-based music therapy has been found to be effective in the treatment of depressive symptoms in people with dementia. ${ }^{33}$ Patients respond better to familiar rather than unfamiliar music. It may be that early on in the development of dementia the patient's musical preferences should be sought and stored for use in reminiscence-based therapy at a later stage.

Commonly music therapy is delivered to groups, but it may be that the music used is not preferred or acceptable to some participants who may only be able to express their dissatisfaction through behavior rather than language. Individual music therapy using a patient's preferred music has been shown to be effective in reducing aggressive behavior. ${ }^{30}$

Music therapy for people with dementia usually involves listening to either recorded or live music, but not participation. No adequate studies could be found of guided improvisation therapy for people with dementia. Guided improvisation therapy involves the playing of a number of tuned and untuned percussion instruments. Through this, the person is able to communicate and express emotion in a relationship with the therapist when normal language and expression are not possible. There is a need for studies of this intervention in dementia where it could be very useful in building a relationship and relieving frustration amongst other symptoms.

The Remington trial of 2002 assigned 68 nursing home residents to single, brief treatments of "soft, calming Baroque classical music", as well as gentle hand massage or a combination of the two. When agitated behaviors were counted during treatments by trained observers, mean agitation fell from baseline by $50 \%$ with music, $37 \%$ with massage, and $61 \%$ with both. These differences were all statistically significant. ${ }^{23}$ The ideals of Baroque music include evoking a consistent and balanced state of mind and rhythmic precision. These characteristics could be beneficial to patients in dementia units, but so far there is little evidence to suggest that Baroque music actually fulfills these ideals.

\section{Our Baroque research}

In our pilot observational study we employed the slow, calm movements of Baroque music by Bach, Handel, Vivaldi, and Corelli piped through a residential home for 3 hours nightly in a ward of 14 people suffering from dementia. ${ }^{34}$ It was a 6-week trial with a 2-week period to collect baseline data, 2 weeks of intervention, then a 2-week washout period. Challenging behaviors were identified and measured in frequency and severity on a devised rating scale. It was the first study in this area of nonpatient-preferred ambient music and yielded strongly positive results, ie, a $40 \%$ reduction in challenging behaviors. The intervention had a profoundly positive effect on one particular subject who would wander, strip herself, and agitate fellow patients. During the intervention period, her behavior improved dramatically. The effect lasted until the intervention was stopped and approached the previous baseline levels for all subjects during the washout period.

The program at this nursing home continued for some time and several nursing homes were interested in employing the intervention based on the limited evidence of this study. One program, at St Hedwig Nursing Home in Blacktown, NSW, Australia, was awarded in 2006 a "Better Practice in Aged Care Award" from the Aged Care Standards and Accreditation Agency Ltd for implementation of a Baroque music program to address challenging behaviors associated with dementia based on the protocol of the pilot study (http://www.accreditation.org.au/accreditation/list-of-awardrecipients/).

Subsequently, we did a randomized controlled study to replicate the findings of the 2003 pilot study in a larger sample. ${ }^{9}$ This study was carried out in a multicultural, dementia-specific, aged-care facility in Newcastle, NSW, with 75 residents. A crossover design with a baselineintervention-washout framework was employed. The results were negative. Some residents were "agitated by the music and some requested it to be turned off". The conclusion was that Baroque music does indeed affect behavior, but in this case negatively, and that if music is to be employed at all, it should be music personally selected by the nursing home 
resident. The suggested reasons for the negative results were that the music was too loud or too fast or that the music patients preferred was different. Our studies are the only ones to exclusively use ambient Baroque music to treat challenging behaviors in nursing home residents.

\section{Conclusion}

Further research will be needed before widespread use of music therapy in the treatment of behavioral symptoms of AD is implemented. O'Connor et al published a systematic review $^{35}$ reporting that only 25 of 118 studies met what they considered a standard quality. The importance of that review is that it provides guidelines to optimize quality standards for future research in this area, including music therapy, patient-preferred music, and the use of ambient Baroque music. Their recommendations include random allocation to distinct control arms of the study; sufficient information about a study's methods to permit replication; larger sample sizes; use of statistical analyses; and, as a matter of stipulation, behavior measures being collected by persons blinded to treatment allocation and to the study's aims. None of the trials cited in this review meet the O'Connor standards. It is recommended that further trials be carried out in this area, adhering to these standards.

Alzheimer dementia is a progressive and common neurodegenerative disease. Behavioral disturbances, including wandering, aggression, psychosis, and apathy are among the most disabling symptoms of this illness. Such symptoms do not respond well to pharmacotherapy, so nondrug interventions are of great interest.

Music is an intervention with proven effects on the human central nervous system, relative low cost, and ease of administration, and little capacity to do harm. Its efficacy is not currently established, but initial studies suggest a role for such therapy as part of a comprehensive plan of management for the behavioral complications of dementia. A more thorough evaluation with the aid of randomized controlled trials is warranted.

Music therapy offers potential benefit on both theoretical and empirical grounds. A distinction between performance and listening must be made, and between live and recorded performance. The use of a program of intervention using a trained music therapist should also be considered a distinct form of music-based intervention. Not surprisingly, music of a type preferred by the patient may be of greater therapeutic benefit than a generic format of music. The relationship between music and behavior in dementia is complex, and symptoms do not always improve with its application.

\section{Acknowledgment}

We thank Steve Mears for the references and his advice.

\section{Disclosure}

The authors report no conflicts of interest in this work.

\section{References}

1. Hebert LE, Weuve J, Scherr PA, Evans DA. Alzheimer disease in the United States (2010-2050) estimated using the 2010 census. Neurology. 2013;80(19):1778-1783.

2. Ferri CP, Prince M, Brayne C, et al. Global prevalence of dementia: a Delphi consensus study. Lancet. 2005;366(9503):2112-2117.

3. Johannesen M, LoGiudice D. Elder abuse: a systematic review of risk factors in community-dwelling elders. Age Ageing. 2013;42(3):292-298.

4. Burton A. Bringing arts-based therapies in from the scientific cold. Lancet Neurol. 2009;8(9):784-785.

5. Vink AC, Birks JS, Bruinsma MS, Scholten RJ. Music therapy for people with dementia. Cochrane Database Syst Rev. 2004;3:CD003477.

6. Opie J, Rosewarne R, O'Connor DW. The efficacy of psychosocial approaches to behaviour disorders in dementia: a systematic literature review. Aust N Z J Psychiatry. 1999;33(6):789-799.

7. Van de Winckel A, Feys H, De Weerdt W, Dom R. Cognitive and behavioural effects of music-based exercises in patients with dementia. Clin Rehabil. 2004;18(3):253-260.

8. Holmes C, Knights A, Dean C, Hodkinson S, Hopkins V. Keep music live: music and the alleviation of apathy in dementia subjects. Int Psychogeriatr. 2006;18(4):623-630.

9. Nair BK, Heim C, Krishnan C, D'Este C, Marley J, Attia J. The effect of Baroque music on behavioural disturbances in patients with dementia. Australas J Ageing. 2011;30(1):11-15.

10. Svansdottir HB, Snaedal J. Music therapy in moderate and severe dementia of Alzheimer's type: a case-control study. Int Psychogeriatr. 2006;18(4):613-621.

11. Sung HC, Chang AM, Lee WL. A preferred music listening intervention to reduce anxiety in older adults with dementia in nursing homes. J Clin Nurs. 2010;19(7-8):1056-1064.

12. Lindenmuth GF, Patel M, Chang PK. Effects of music on sleep in healthy elderly and subjects with senile dementia of the Alzheimer type. Am J Alzheimers Dis Other Demen. 1992;7(2):13-20.

13. Lai HL, Good M. Music improves sleep quality in older adults. $J A d v$ Nurs. 2005;49(3):234-244.

14. Raglio A, Bellelli G, Traficante D, et al. Efficacy of music therapy in the treatment of behavioral and psychiatric symptoms of dementia. Alzheimer Dis Assoc Disord. 2008;22(2):158-162.

15. Suzuki M, Kanamori M, Nagasawa S, Tokiko I, Takayuki S. Music therapy-induced changes in behavioral evaluations, and saliva chromogranin A and immunoglobulin A concentrations in elderly patients with senile dementia. G Geriatr Gerontol Int. 2007;7(1):61-71.

16. Ho YC, Cheung MC, Chan AS. Music training improves verbal but not visual memory: cross-sectional and longitudinal explorations in children. Neuropsychology. 2003;17(3):439-450.

17. Chan AS, Ho YC, Cheung MC. Music training improves verbal memory. Nature. 1998;396(6707):128.

18. Sridharan D, Levitin DJ, Chafe CH, Berger J, Menon V. Neural dynamics of event segmentation in music: converging evidence for dissociable ventral and dorsal networks. Neuron. 2007;55(3):521-532.

19. Hailstone JC, Omar R, Warren JD. Relatively preserved knowledge of music in semantic dementia. J Neurol Neurosurg Psychiatry. 2009;80(7):808-809.

20. Vanstone AD, Cuddy LL. Musical memory in Alzheimer disease. Neuropsychol Dev Cogn B Aging Neuropsychol Cogn. 2010;17(1): 108-128.

21. Baird A, Samson S. Memory for music in Alzheimer's disease: unforgettable? Neuropsychol Rev. 2009;19(1):85-101. 
22. Brotons M, Pickett-Cooper PK. The effect of music therapy intervention on agitation behaviors of Alzheimer's disease patients. J Music Ther. 1996;33(1):2-18.

23. Remington R. Calming music and hand massage with agitated elderly. Nurs Res. 2002;51(5):317-323.

24. Groene RW. Effectiveness of music therapy 1:1 intervention with individuals having senile dementia of the Alzheimer's type. J Music Ther. 1993;30(3):138-157.

25. Pollack NJ, Namazi KH. The effect of music participation on the social behavior of Alzheimer's disease patients. J Music Ther. 1992;29(1):54-67.

26. Dubois JM, Bartter T, Pratter MR. Music improves patient comfort level during outpatient bronchoscopy. Chest. 1995;108(1):129-130.

27. Bampton P, Draper B. Effect of relaxation music on patient tolerance of gastrointestinal endoscopic procedures. J Clin Gastroenterol. 1997;25(1):343-345.

28. Byers J, Smyth K. Effect of a music intervention on noise annoyance, heart rate, and blood pressure in cardiac surgery patients. Am J Crit Care. 1997;6(3):183-191.
29. Kneafsey R. The therapeutic use of music in a care of the elderly setting a literature review. J Clin Nurs. 1997;6(5):341-346.

30. Clark ME, Lipe AW, Bilbrey M. Use of music to decrease aggressive behaviors in people with dementia. J Gerontol Nurs. 1998;24(7):10-17.

31. Pacchetti C, Aglieri R, Mancini F, Martignoni E, Nappi G. Active music therapy and Parkinson's disease: methods. Funct Neurol. 1998;13(1):57.

32. Casby JA, Holm MB. The effect of music on repetitive disruptive vocalizations of persons with dementia. Am J Occup Ther. 1994;48(10): 883-889.

33. Ashida S. The effect of reminiscence music therapy sessions on changes in depressive symptoms in elderly persons with dementia.J Music Ther. 2000;37(3):170-182.

34. Heim C, Nair BK, Mowbray D, Tavender J. Effects of ambient baroque music on patients with dementia. Australas J Ageing. 2003;22(4): $211-212$.

35. O’Connor DW, Ames D, Gardner B, King M. Psychosocial treatments of psychological symptoms in dementia: a systematic review of reports meeting quality standards. Int Psychogeriatr. 2009;21(2):241-251.
Degenerative Neurological and Neuromuscular Disease

\section{Publish your work in this journal}

Degenerative Neurological and Neuromuscular Disease is an international, peer-reviewed, open access journal focusing on research into degenerative neurological and neuromuscular disease, identification of therapeutic targets and the optimal use of preventative and integrated treatment interventions to achieve improved outcomes, enhanced

\section{Dovepress}

survival and quality of life for the patient. The manuscript management system is completely online and includes a very quick and fair peer-review system. Visit http://www.dovepress.com/testimonials.php to read real quotes from published authors.

Submit your manuscript here: http://www.dovepress.com/degenerative-neurological-and-neuromuscular-disease-journal 